DOI: 10.3901/JME.2021.15.222

\title{
仿皮肤三维多孔点阵结构压缩吸能性能研究
}

\author{
纪小刚 ${ }^{1,2}$ 张建安 $^{1}$ 栾宇豪 $^{1}$ 张溪溪 $^{1}$ 胡海涛 $^{1}$ \\ (1. 江南大学机械工程学院 无锡 214122; \\ 2. 江南大学江苏省食品先进制造装备技术重点实验室 无锡 214122)
}

\begin{abstract}
摘要：基于 DLP 数字光处理(Digital light processing, DLP) 3D 打印技术，制造出具有规则多孔特点的三维多孔点阵结构，以 使其具有较宽压缩应力平台的特点。研究和设计了 3 种单元网格构型细胞, 并由此组合成不同的规则多孔点阵结构, 采用特 定配比方式的光固型柔性树脂材料制造成型; 根据仿生设计原理, 分析皮肤纵切面结构特点, 基于试验数据并结合皮肤结构, 找到最优三维多孔结构, 以获得良好的压缩应力平台; 该三维多孔结构具有规则可控的优势, 并结合适当的软质材料, 表现 出了良好的机械性能。该研究成果具有较为广阔的发展空间, 其结构可用于组织工程皮肤体外构件技术中的三维支架材料, 也可作为振动和能量吸收领域的优质缓冲或阻尼材料。
\end{abstract}

关键词: 单元网格构型; 应力平台; 点阵结构; 3D 打印

中图分类号: TH122

\section{Research on Compression Energy Absorption Performance of Skin-like 3D Porous Lattice Structure}

\author{
JI Xiaogang ${ }^{1,2}$ ZHANG Jianan $^{1} \quad$ LUAN Yuhao $^{1} \quad$ ZHANG Xixi $^{1} \quad$ HU Haitao $^{1}$ \\ (1. School of Mechanical Engineering, Jiangnan University, Wuxi 214122; \\ 2. Jiangsu Key Laboratory of Advanced Food Manufacturing Equipment and Technology \\ (Jiangnan University), Wuxi 214122)
}

\begin{abstract}
A three-dimensional porous lattice structure with regular porous characteristics based on digital light processing (DLP) 3D printing technology is manufactured, so that it has the characteristics of a wider compressive stress platform. Three cell grid configurations are studied and designed, and thus are combined into different regular three-dimensional porous lattice structures, which are manufactured by using light solid flexible resin material with a special ratio. According to the principle of bionic design, the structural characteristics of the skin longitudinal section are analyzed. Based on the experimental data and combined with the skin structure, Find the optimal three-dimensional porous structure to obtain a good compressive stress platform. The three-dimensional porous structure has the advantages of regular controllability, combined with appropriate soft materials, and shows good mechanical properties. The research results have a relatively broad Development space, whose structure can be used as a three-dimensional scaffold material in the tissue engineering skin external component technology, and can also be used as a high-quality buffer or damping material in the field of vibration and energy absorption.
\end{abstract}

Key words: cell grid configuration; stress platform; lattice structure; 3D printing

\section{0 前言}

近年来, 三维多孔点阵结构因其具有优良的结 构效率和广泛的多功能应用前景, 成为众多研究的 热点。

20200819 收到初稿, 20210218 收到修改稿
针对单元网格构型，1995 年，SIGMUND 等 ${ }^{[1]}$ 提出了四种典型的单元网格结构, 并针对这几种结 构进行了相应的弹性性能研究。该结构具备较强的 强度和刚度, 更重要的是还在一定程度上降低了材 料的损耗。另外，空间点阵结构是一种新型的、国 际上公认非常有前景的新一代轻质结构, 于 2000 年由哈佛大学的 ASHHY 教授和 MIT 的 GIBSON 教 授等率先提出。这种规则多孔微观结构, 具有很强 
的能量吸收以及传热和渗透等特殊性能, 在航空航天、 机械以及医疗领域都有着非常广泛的应用前景 ${ }^{[2-3]}$ 。

目前, 国外在多孔点阵结构的研究主要集中在 金属材料方面, 研究其点阵结构的力学特性以及相 关成型工艺 ${ }^{[4-7]}$ 。在国内, 以北京大学方岱宁课题组 为代表的研究人员已经在树脂基及炭/炭平板行空 间点阵结构的制备、性能表征以及力学性能的设计 与分析等方面开展了大量的工作 ${ }^{[8-9]}$ 。研究表明该空 间点阵结构在力学特性上具有高比强度、高比刚度、 剪切强度等优点。

本文试图以单元网格构型设计为基础, 在空间 进行 $X 、 Y 、 Z$ 三个方向的点阵拓展, 设计一种规则 三维多孔点阵结构。其创新点就在于将单元网格构 型和空间点阵结构相结合, 并参考皮肤模量进行材 料设计, 选用软质的柔性树脂作为材料进行 3D 打 印成型, 最终形成多孔点阵结构。由此可制造出具 有较宽压缩应力平台的弹性多孔结构, 并结合一系 列微观参数以及结构的调整来改变所设计的点阵结 构。本文基于 ANSYS 仿真进行初步分析, 研究多 孔点阵结构受压力学性能, 进而通过单轴压缩试验 进行验证以及辅助设计, 从而找到具有良好压缩吸 能性能的最优解。

\section{1 单元网格构型设计}

\section{1 柔性树脂材料性能测试}

均匀材质的力学特性对于皮肤并不适用, 皮肤 并无特定的单一弹性模量, 其力学性能是根据皮肤 所受外界载荷的变化而变化。以人体为例, 不同部 位的皮肤力学性能也截然不同。本文拟选取与皮肤 模量相近似的材料作为模型材料, 进行相关力学特 性的研究。

模量和泊松比可以描述皮肤的弹性行为, 其作 为皮肤力学性能最重要的参数之一, 已经得到广泛 的测量 ${ }^{[10]}$, 表 1 简单列举了人体不同部位的皮肤弹 性模量。

表 1 皮肤弹性模量

\begin{tabular}{cccc}
\hline 皮肤试样 & 测量值 $/ \mathrm{MPa}$ & 方法 & 年龄 \\
\hline 大腿 & 0.84 & 拉伸 & $22 \sim 68$ 岁 \\
前臂 & 0.14 & 抽吸 & 25 岁前 \\
\hline
\end{tabular}

设计的点阵结构所选材料为光固型柔性树脂, 这种柔性树脂主要由四种材料聚合而成, 分别为 7162 弹性单官聚氨酯丙烯酸树脂、3906 弹性双官聚 氨酯丙烯酸树脂、 $\mathrm{ACMO}$ 和 IBOA。由于这种柔性
树脂是根据试验需求进行配比的, 其力学性能未知。 为了获得该柔性树脂的各个力学性能参数, 本文参 照 ISO3167 将柔性树脂制成 1A 型标准拉伸试样, 并在万能试验机上进行试验。由于 3D 打印机尺寸 限制, 将原先标准尺寸按 1:2 比例进行缩放建模且 厚度不变进行打印成型, 拉伸试样如图 1 所示。由 拉伸试验测得该柔性树脂所能承受的最大载重平均 值为 $31.42 \mathrm{~N}$, 分析计算可得其弹性模量为 0.342 $\mathrm{MPa}$, 与人体皮肤 $0.5 \mathrm{MPa}$ 的弹性模量相差不大 ${ }^{[9]}$ 。

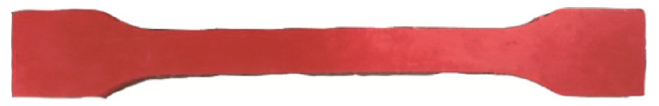

图 1 ISO3167 标准试样

\section{2 单元网格构型设计}

本文的目的是设计一种微型且规则的三维 多孔点阵结构。将单元网格的外接正方体边长设 为 $2.4 \mathrm{~mm}$, 内部支撑结构采用圆柱形结构。在 研究多孔结构的力学性能时, 其每个单元胞体结 构的相对密度也是一个较为关键的因素(单元网 格的相对密度是指网格相对原有实心结构的体 积比)。因此在设计之前需要确定几种单元网格 构型的相对密度。所设计的 3 种单元网格构型各 尺寸以及相对密度见表 2 。

\section{表 2 单胞尺寸及相对密度}

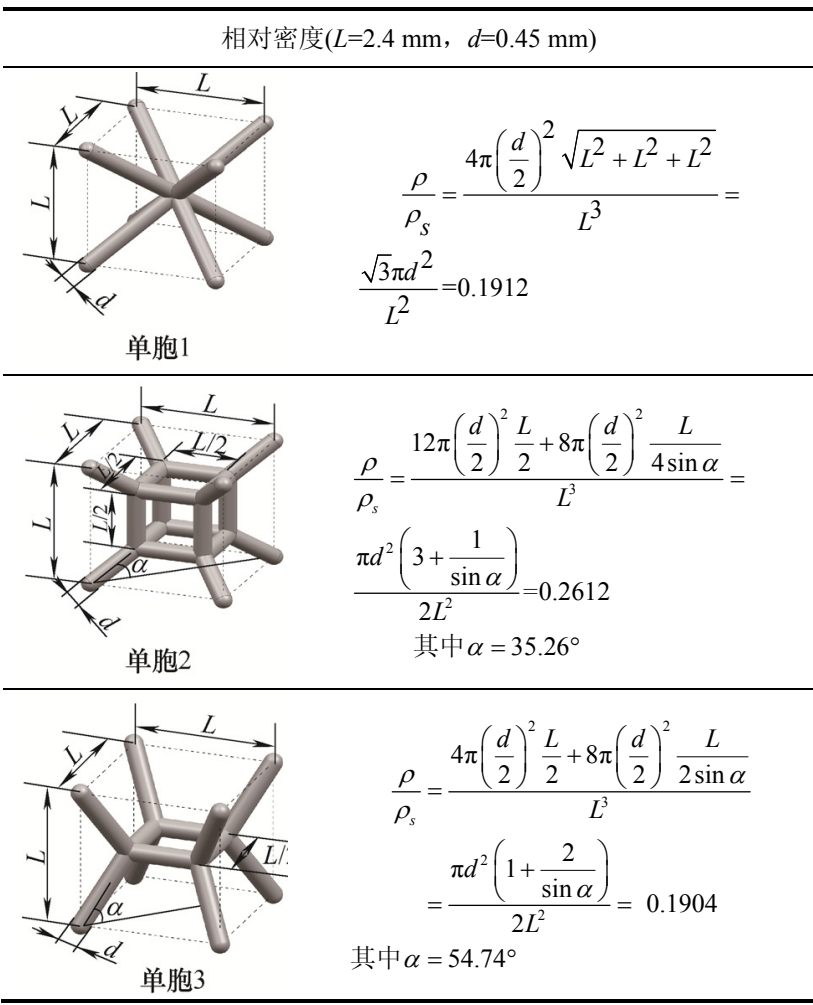

\section{3 三维多孔点阵结构设计}

单元网格构型确定后, 即可进行三维多孔点阵 
结构的设计。该点阵结构内部的 “芯” 是相对应单 胞结构分别向 $X 、 Y 、 Z$ 三个坐标轴方向进行线性阵 列所得, 形成由 50 个单胞拼接而成的结构。双层胞 体尺寸为 $12 \mathrm{~mm} \times 12 \mathrm{~mm} \times 4.8 \mathrm{~mm}$, 同时底部加入一 块尺寸为 $12.8 \mathrm{~mm} \times 12.8 \mathrm{~mm} \times 0.8 \mathrm{~mm}$ 的底板。最终 得到的三种单胞组成的三维多孔点阵结构如图 2 所示。

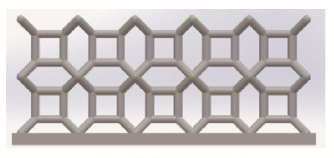

(a) 点阵结构 1

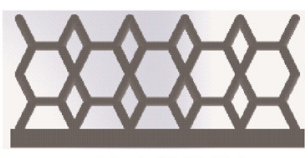

(b) 点阵结构 2

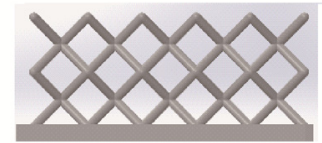

(c) 点阵结构 3

图 2 三种多孔点阵结构

\section{4 结构初步打印试验}

由于本文是对一种微小且规则排列的多孔结构 进行研究, 且该多孔结构十分复杂, 想要实现软质 材料的 3D 打印比较困难。主要问题在于单胞杆件 尺寸过细, 在打印过程中容易发生断裂。同时, 由 于单胞与单胞之间的孔隙过小, 容易出现堵塞。经 过各类材料以及成形工艺的试验, 最终找到了成型 的最佳方案：材料选择一种具有一定柔度的光敏树 脂, 成型技术选择 DLP 3D 打印。该光敏树脂是由 7162 弹性单官聚氨酯丙烯酸树脂和 3906 弹性双官 聚氨酯丙烯酸树脂按一定比列混合而成。打印成型 结果如图 3 所示。

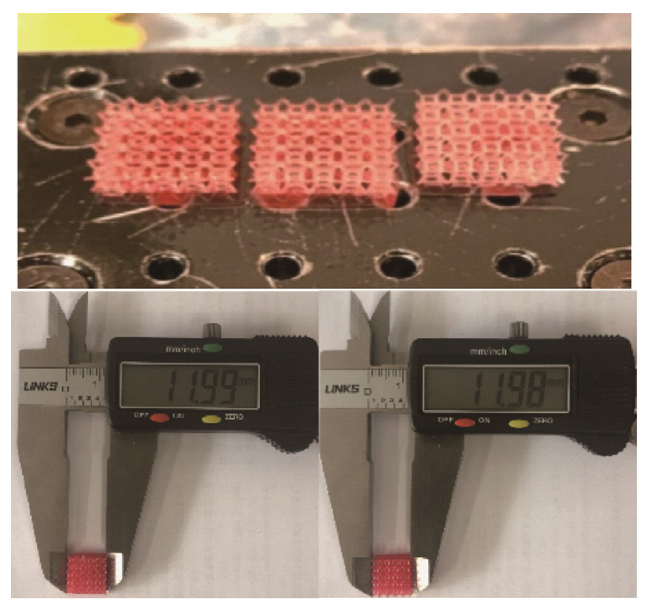

图 3 打印成果图

\section{2 三维多孔结构有限元分析及试验}

有限元初步分析表明, 该结构在压缩载荷下会
产生明显的弹性屈曲, 该结构可出现较好的压缩 应力平台, 原因在于压缩期间沿 $X-Y$ 平面的两个 横向方向单元的双弹性屈曲。这种类似蜂窝状的 固体结构受压后, 当变形超过线性弹性状态时, 通过利用细胞结构的大变形和塌陷来实现能量吸 收, 在应力-应变曲线中表现出来的就是中间平台 段部分。

\section{1 双层三维多孔点阵结构压缩性能分析}

本节对图 2 中三种由不同单胞阵列构成的点 阵结构 1、2、3 分别进行 ANSYS 有限元压缩仿 真分析, 并对其打印模型进行压缩试验, 其分析 的区域主要由两层阵列的单胞组成。本节只对三 种单胞组成的点阵结构进行分析, 研究这三种单 胞的力学特性, 单胞杆件直径设为 $0.45 \mathrm{~mm}$ 。由 于选用的材料为一种柔性的光敏树脂, 并且该多 孔结构为孔隙率较大的一种结构, 在进行单轴压 缩时整体可以实现较大的变形。有限元分析所用 的模式为添加位移加载, 为了使其达到较好的致 密化效果, 将位移加载设置为 $-3 \mathrm{~mm}$, 即压缩 $62.5 \%$ 。模拟区域分成两块, 其中主要承载区由双 层共计 50 个单元胞体结构组成, 下板部分保持固 定约束。由于在后续的单轴压缩试验中, 不能很 好的观察到结构在受压时及受压后的真实情况。 本文将给出模型有限元仿真的变形及应力云图, 以便直观地观察出每个杆件的真实应力状况以及 各节点的受力情况, 有助于后续的分析研究, 应 力-应变如图 4 6 所示。
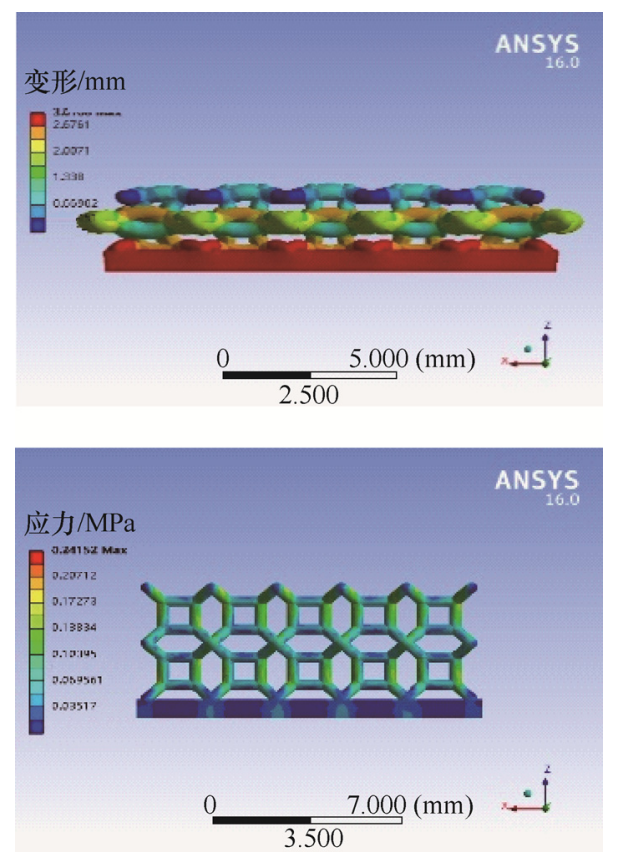

图 4 1-0.45 变形及应力云图 

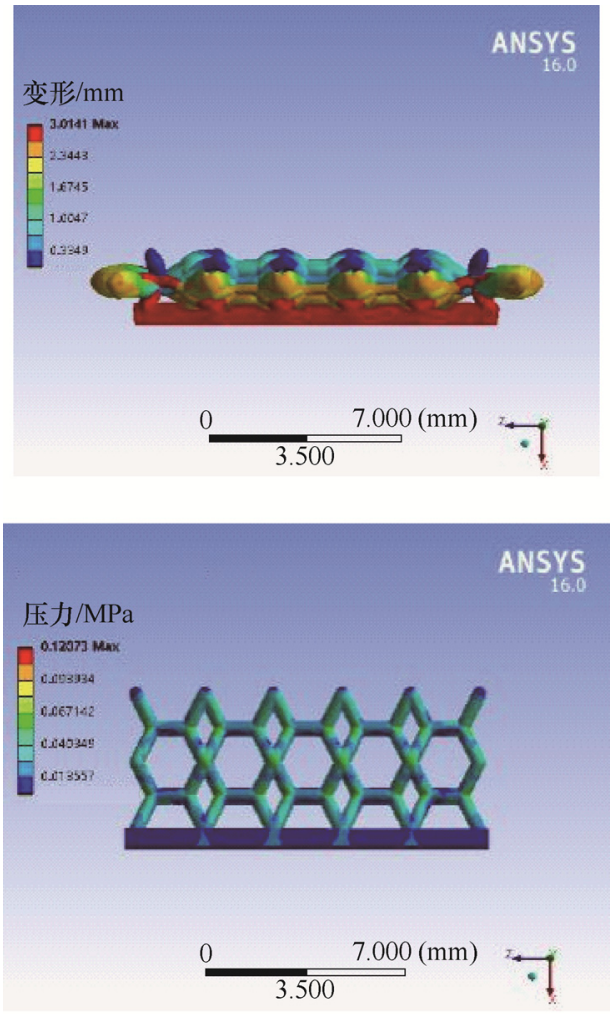

图 5 2-0.45 变形及应力云图
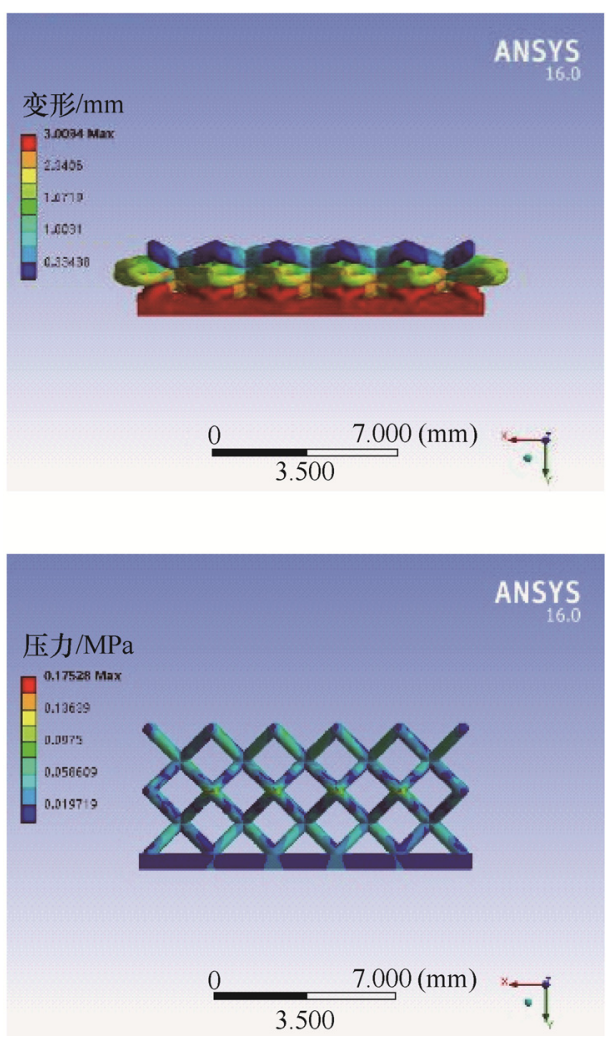

图 6 3-0.45 变形及应力云图

为了验证有限元模拟的结果是否正确, 本文打 印了上述三种点阵结构并对其进行单轴压缩试验。 模型从打印底板上取下后, 将其放入 100\%的工业酒 精中进行超声波除杂并除去结构表面附着的残余树
脂, 再放入 $U V$ 箱中进行二次固化 $5 \mathrm{~min}$, 最后置于 室温下 $24 \mathrm{~h}$ 。模型后处理结束后, 进行 $\mathrm{Z}$ 轴方向的 单轴压缩试验, 使用的试验器材是 CTM2500 万能 材料试验机 (最大荷重: $10 \mathrm{KN}$ 荷重精度 $\pm 0.01 \%$; 精 度等级: 0.5 级; 位移分辨率: $0.03 \mu \mathrm{m}$ ), 装置如 图 7 所示。

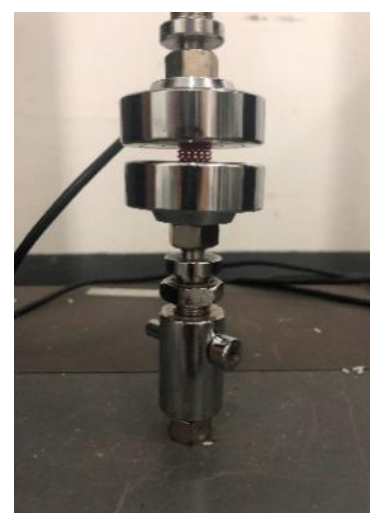

图 7 压缩试验装置图

图 8 给出了三种点阵结构在 $Z$ 轴压缩载荷作用 下, 有限元仿真以及试验所得的应力-应变曲线的对 比图, 虚线处为有限元仿真结果的平台应力值。虽 然试验和仿真应力-应变曲线的整体趋势基本相似, 但是在相对应的值上存在偏差。对于平台应力, 试 验结果与模拟仿真结果最大相差 $24 \%$, 仿真的结果 都略大于试验结果。造成该误差的原因主要有以下 几点: (1) 打印设备精度的限制; (2) 模型在进行二 次清洗时杆件上所残留的树脂未清洗干净从而造成 部分孔隙堵塞, 导致结构实际相对密度与理论存在 偏差。同时, 在添加载荷后, 某些节点受力不均导 致平台应力产生波动, 但该误差在允许的范围之内。 因此, 后续将以试验所得的应力-应变曲线为主进行 相关分析研究。

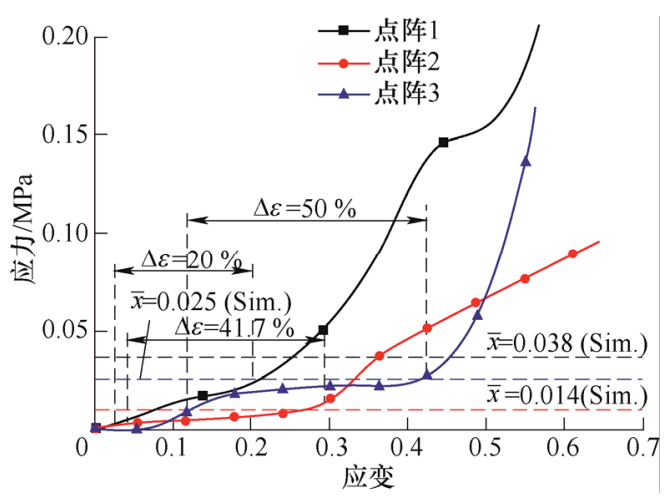

图 8 三种点阵结构压缩应力-应变曲线

本文选用的材料具有各向同性的特点, 因此结 合图 4 6, 可以看出在该结构受压时, 整体变形状 态是一种对称的状态, 应力的主要集中区域出现在 
结构各杆件的连接处以及与平面呈一定倾斜角的杆 件处。

显然, 可将图 8 试验所得的应力-应变曲线分为 三项, 第一项描述的是材料在弹性变形段, 第二项 描述的是其内部胞体逐渐坞塌段 (或应力平台段), 第三项则是内部胞体完全坞塌后产生致密化效 应段。

根据应力-应变曲线图, 发现点阵 1 结构的承载 能力较其他两种结构强许多, 在相同位移加载下其 应力峰值较高。但该结构是由一种柔性树脂制成, 整体结构十分软, 所以在这项研究中并不关注其承 载能力, 而更加关注其在受压时内部结构反应出的 力学特性, 也就是吸能应力平台 ${ }^{[11]}$, 将这一平台段 的占比用 $\Delta \varepsilon$ 进行定义, 即吸能平台占用率。

表 3 给出了上述三种点阵结构的相对密度以 及应力平台的占用率, 可以看出相对密度较高的 点阵 1 模型的平台占用率与点阵 2 和 3 相差较大。 因其相对密度较高, 相应的孔隙率较低, 从而导 致可压缩的空间变小, 使其较早地进入致密化效 应段。从其应力一应变曲线可以看出, 同应变的状 态下表现出了较高的应力。相较点阵 2 和点阵 3 模型, 点阵 3 相对密度高于点阵 2 , 表现出了较 好的应力平台。

表 3 双层点阵结构应力平台占用率及相对密度

\begin{tabular}{cccc}
\hline 模型号 & 相对密度 & 应力 $/ \mathrm{MPa}$ & 占用率 $\Delta \varepsilon(\%)$ \\
\hline 点阵 1 & 0.2612 & 0.05 & 20.00 \\
点阵 2 & 0.1904 & 0.02 & 41.67 \\
点阵 3 & 0.1912 & 0.05 & 50.00 \\
\hline
\end{tabular}

这也引起了本文对点阵 3 模型的关注, 点阵 3 模型其实就是俗称的金字塔点阵结构 ${ }^{[12-13]}$ 。后续的 研究, 将在不改变点阵 3 结构的基础上, 探究胞体 杆件直径对结构压缩力学性能的影响。

\section{2 点阵 3 结构不同胞体杆件直径压缩性能分析}

本文研究初期, 发现每个点阵结构的弹性屈曲 与单胞的杆件直径有着密切的关系。通过第 2.1 节 的研究, 发现点阵 3 这种金字塔形单胞组成的点阵 结构在较高的相对密度下，受压后可以保持良好的 应力平台, 所以本节针对该单一结构对其进行杆件 直径参数的研究。

本文主要研究的是微小层面的三维多孔结构, 同时又考虑到现有的工艺能否实现后期的 3D 打印 成型以及压缩试验。将其直径参数初步定为 $0.35 \mathrm{~mm} 、 0.40 \mathrm{~mm} 、 0.45 \mathrm{~mm} 、 0.50 \mathrm{~mm}$ 这四种, 来
研究其对规则三维多孔点阵结构压缩性能的影响, 压缩应力-应变曲线如图 9 所示。

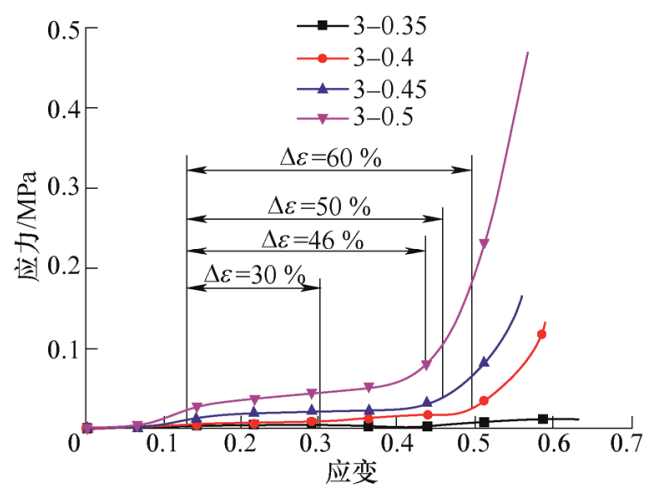

图 9 点阵 3 不同杆件直径压缩应力-应变曲线图

如表 4 所示，平台应力随着杆件直径增大而增 大。但是平台应力占用率并不随着杆件直径大小呈 线性进行变化, 反而直径为 $0.35 \mathrm{~mm}$ 时占用率最小。 在 $X-Y$ 成型面上, 发现打印出来的模型, 杆件的实 际直径并不是理论设计的直径尺寸，材料在胞体杆 件上会存在着一些粘连，如图 10 所示。

\section{表 4 不同杆件直径点阵结构应力平台占用率及相对密度}

\begin{tabular}{cccc}
\hline 直径 $/ \mathrm{mm}$ & 相对密度 & 应力 $/ \mathrm{MPa}$ & 占用率 $\Delta \varepsilon(\%)$ \\
\hline 0.35 & 0.1157 & 0.020 & 30 \\
0.40 & 0.1511 & 0.022 & 63 \\
0.45 & 0.1912 & 0.032 & 50 \\
0.50 & 0.2360 & 0.052 & 48 \\
\hline
\end{tabular}

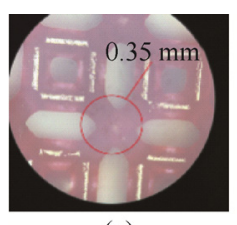

(a)

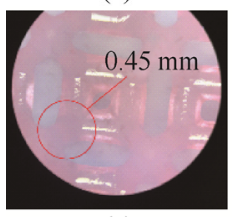

(c)

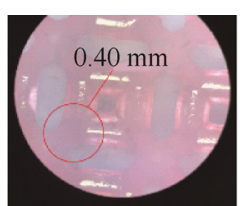

(b)

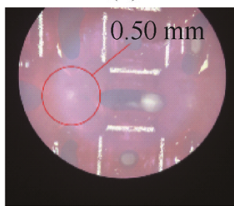

(d)
图 10 各胞体杆件直径 50 倍显微镜结构

由于这种现象的存在, 使得实际模型的相对密 度与理论计算值会存在一定的误差。通过综合比较, 当胞体杆件直径为 $0.45 \mathrm{~mm}$ 时, 从其平台应力值、 占用率以及打印模型各孔隙之间的成型状况来看, 都表现出了良好的压缩性能。因此, 本文着重在胞 体杆件直径为 $0.45 \mathrm{~mm}$ 的尺寸上进行不同拓扑结构 的研究。 


\section{3 不同拓扑结构的点阵结构压缩性能分析}

根据前两节的研究, 发现这 3 种单胞阵列的双 层点阵结构都表现出了良好的压缩性能。其中单胞 3 组成的点阵结构的压缩应力平台最为良好。

本文设想, 通过不同的拓扑结构研究该三维多孔 点阵结构的压缩力学性能。此节将单胞 $1 、 2 、 3$ 模型 分别先在 $X 、 Y$ 方向进行阵列, 形成单层点阵结构编号 为 $1 、 2 、 3$ 。再将这三种单层点阵结构进行不同的排 列组合, 形成双层点阵结构, 其设计结构如图 11 所示。 压缩仿真变形、应力云图如图 12 14 所示。

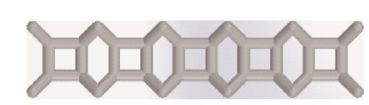

(a) 单层点阵1

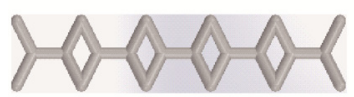

(b) 单层点阵 2

(c) 单层点阵3

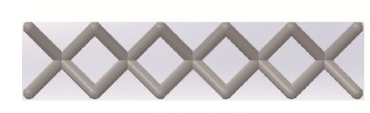

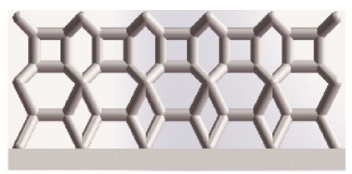

(d) 点阵1-2

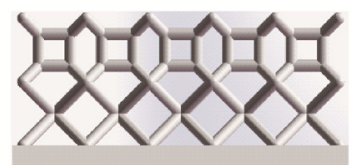

(e) 点阵1-3

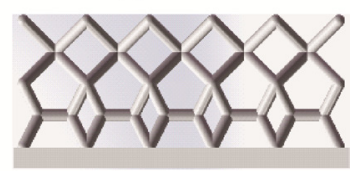

(f) 点阵2-3
图 11 双层拓扑结构
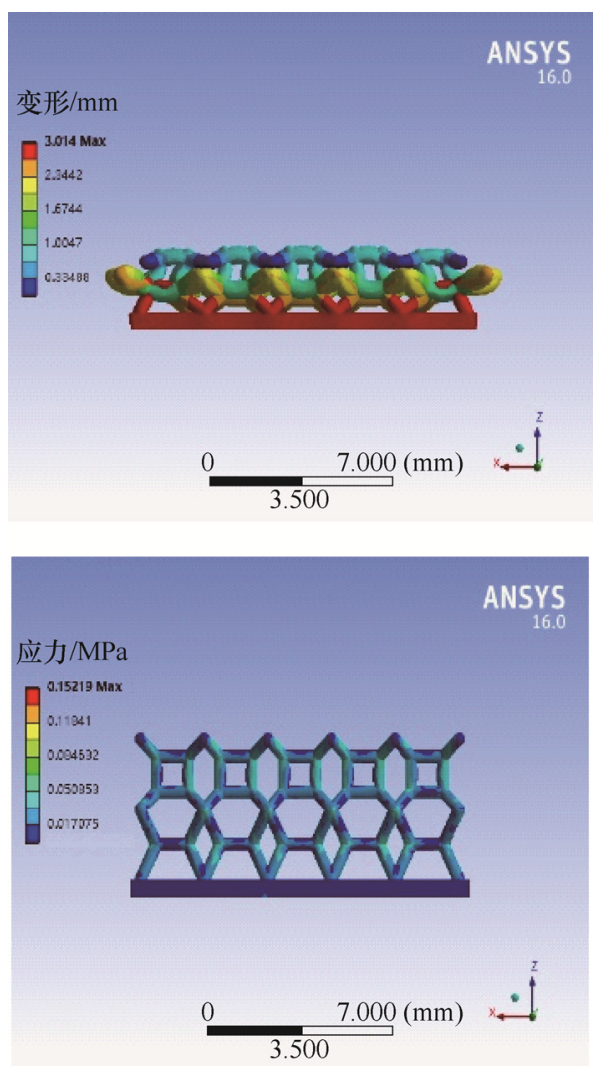

图 12 点阵 1-2 变形及应力云图
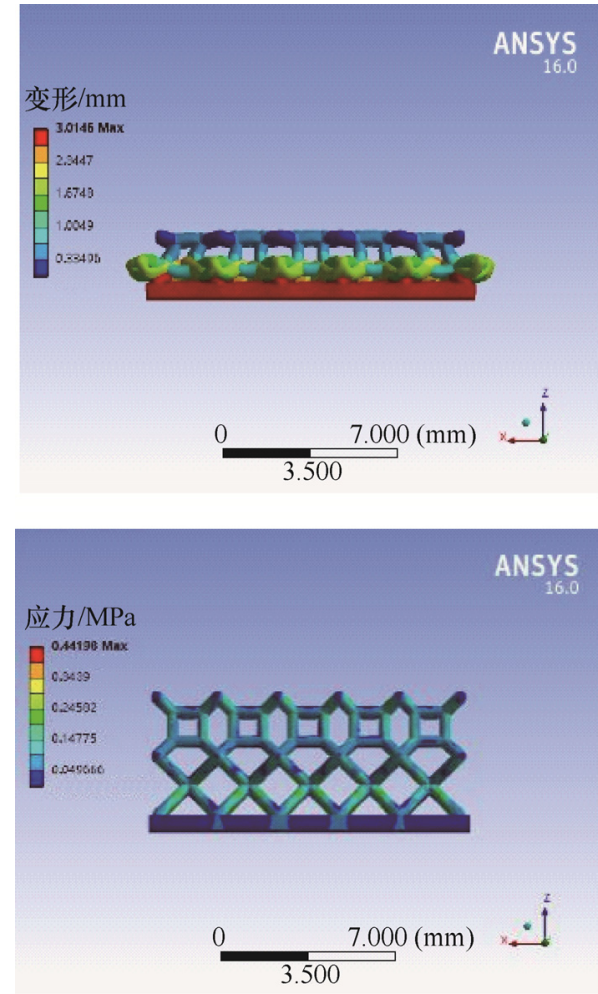

图 13 点阵 1-3 变形及应力云图
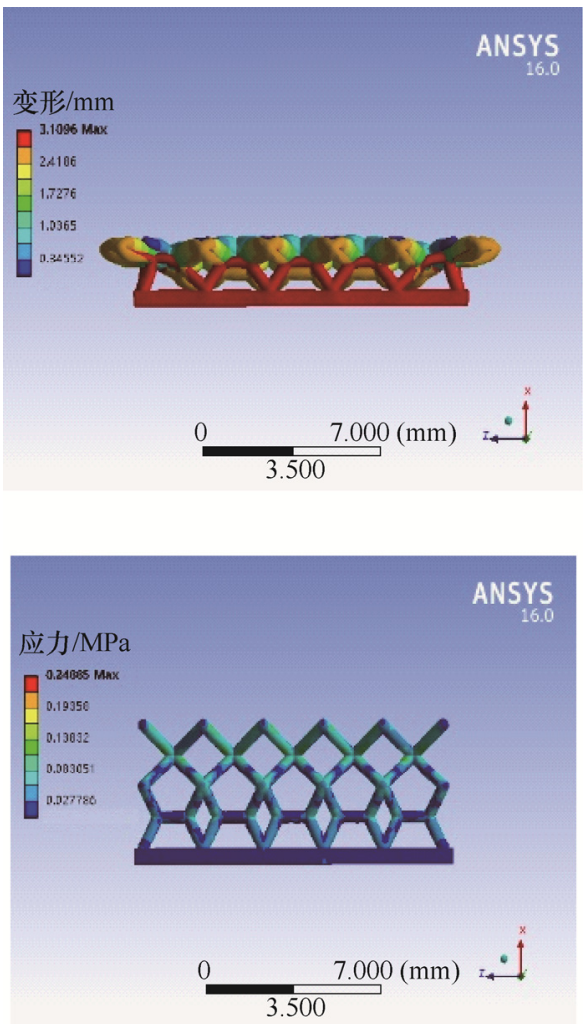

图 14 点阵 2-3 变形及应力云图

根据上述仿真云图, 当垂直方向上进行 $-3 \mathrm{~mm}$ 位移加载完毕后, 从图上可以清晰地看出, 拥有点 阵 1 的结构在加载完毕后都未产生完全的致密化, 如点阵 1-2、点阵 1-3 所示。压缩试验打印模型如 图 15 所示, 压缩应力-应变曲线如图 16 所示, 分析 


\section{结果见表 5 。}

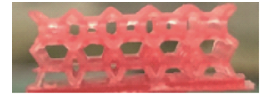

(a) $1-2$

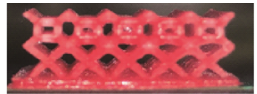

(b) 1-3

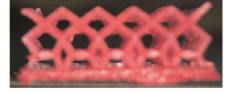

(c) 2-3
图 15 拓扑结构打印成品图

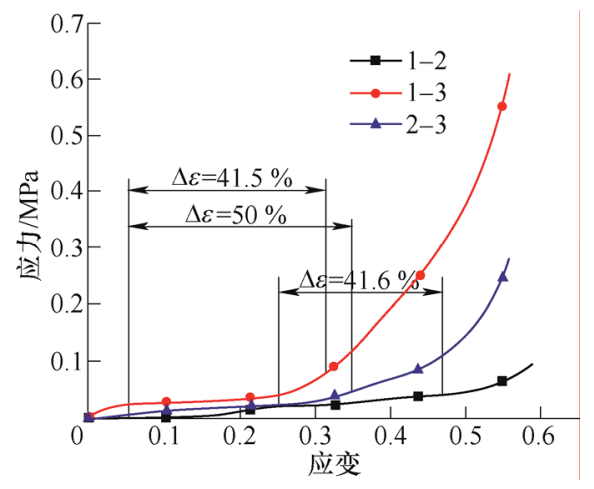

图 16 拓扑结构压缩应力-应变曲线

表 5 拓扑点阵结构应力平台占用率及相对密度

\begin{tabular}{cccc}
\hline 结构 & 相对密度 & 应力 $/ \mathrm{MPa}$ & 占用率 $\Delta \varepsilon(\%)$ \\
\hline $1-2$ & 0.2258 & 0.033 & 41.6 \\
$1-3$ & 0.2262 & 0.045 & 41.5 \\
$2-3$ & 0.1908 & 0.053 & 50.0 \\
\hline
\end{tabular}

结合变形云图以及应力-应变曲线发现, 拥有 点阵 2 的拓扑结构初始应力值较小以及能更好的 进行细胞坍塌从而实现结构的吸能, 所以从应力应变曲线上反应出的就是, 较晚的进入致密化的 应力上升期, 但同时也会较晚的进入吸能平台段。 与其相反, 点阵 1-3 可较早的进入平台吸能段, 从应力-应变图上反应出的就是, 受压初期点阵 1-3 结构的应力会迅速上升, 使其较快的达到平 台应力值。

由表 5 的数值可以看出, 本文设计的拓扑结构 在平台应力值上较之前有了一定的提升。同时, 在 保证了一定的应力平台占用率的前提下, 使结构仍 然存在着一定的孔隙。

\section{3 仿皮肤多孔点阵结构压缩性能分析}

\section{1 皮肤纵切面结构分析}

如图 17 所示, 表皮层主要是由角质形成细胞 组成, 表皮层外部都分化成角质层, 密度较大, 真皮层上层纤维细胞密度较高, 低层密度较低。 因此, 可以把皮肤切面结构看成一个密度由高到 低的梯度结构。本文设想将三维多孔点阵结构设 计成一个仿皮肤的三层结构, 相对密度由高到低
进行排列。

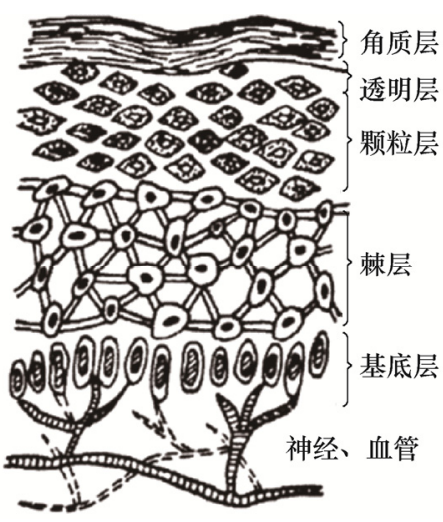

图 17 皮肤切面结构 ${ }^{[14]}$

本节对三维多孔点阵结构进行仿皮肤梯度结构 设计, 如图 18 所示, 并研究其压缩吸能平台的特点, 压缩变形云图、应力云图如图 19 20 所示。同时将 这三层结构进行不同方式的排列, 并与梯度结构进 行压缩性能的比较。

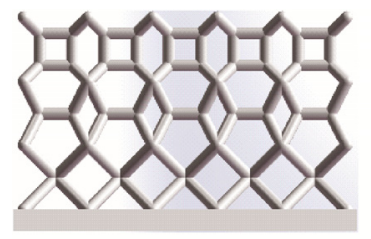

(a) 1-2-3仿皮肤度设计

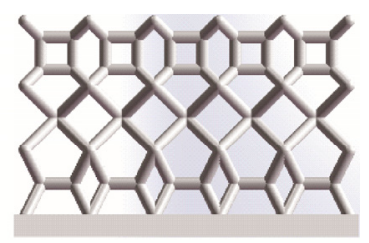

(b) 1-3-2三层拓扑结构设计
图 18 不同排列的三层三维多孔点阵模型
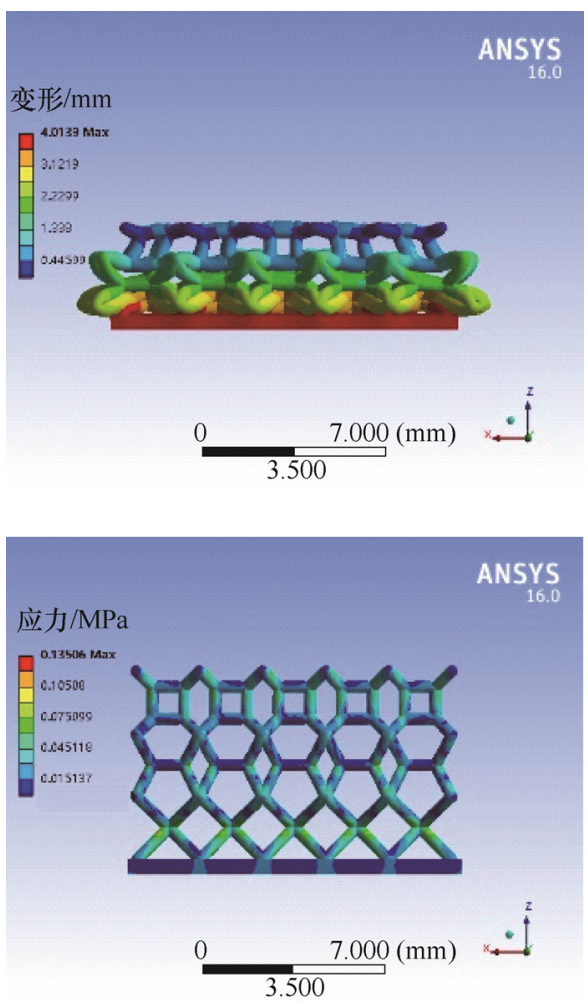

图 19 1-2-3 变形及应力云图 

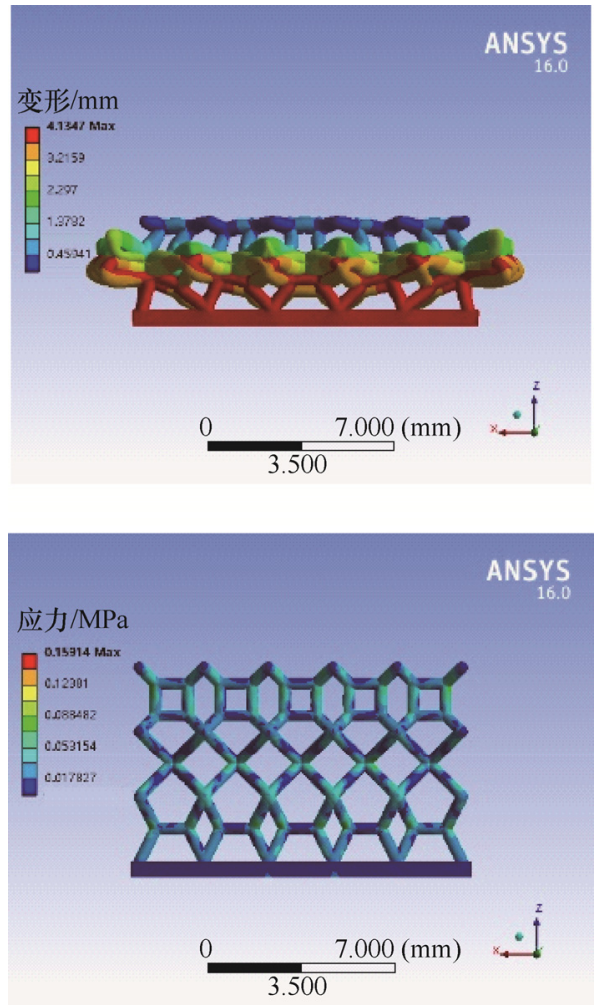

图 $20 \quad 1-3-2$ 变形及应力云图

由于该结构是三层点阵结构, 为了保证其与之 前的两层拓扑结构实现相同的压缩量, 即 $65 \%$, 因 此给定位移载荷为 $-4 \mathrm{~mm}$ 。三层点阵结构打印成品 图如图 21 所示, 压缩应力-应变曲线如图 22 所示。

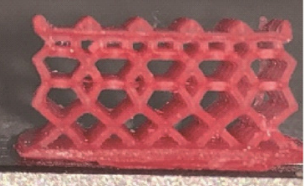

(a) 1-2-3

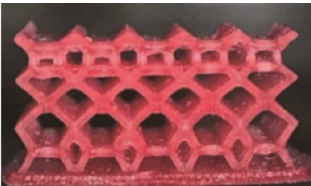

(b) $1-3-2$
图 21 三层点阵结构打印成品图

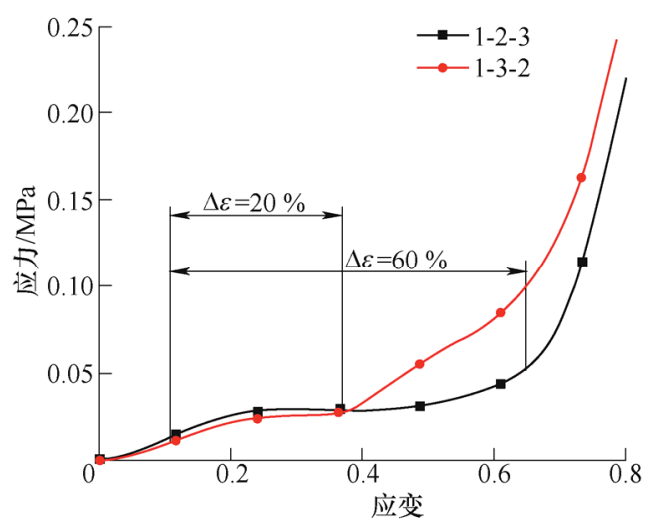

图 22 三层点阵结构应力-应变曲线

由图 19、20 可以看出, 点阵 1 作为第一层最先 受压的区域, 在加载完毕后并未完全达到致密化。 这主要是由于其胞体在 $Z$ 轴方向上有与其平行的杆 件能承受一定的弹性屈曲, 将载荷传递到下方结构
后使得下方结构率先致密化。

由于该三层拓扑结构是由点阵 1、2、3 进行不 同排列方式组合而成的，所以其相对密度是一致的。 点阵 1-2-3 是根据皮肤纵切面结构进行的有关相对 密度的梯度设计，由表 6 所示，点阵 1-2-3 结构表 现出良好的应力平台, 其占用率高达 $60 \%$, 而点阵 1-3-2 结构却只有 20\%。

\section{表 6 三层点阵结构应力平台占用率及相对密度}

\begin{tabular}{cccc}
\hline 结构 & 相对密度 & 应力 $/ \mathrm{MPa}$ & 占用率 $\Delta \varepsilon(\%)$ \\
\hline $1-2-3$ & 0.2143 & 0.031 & 20 \\
$1-3-2$ & 0.2143 & 0.025 & 60 \\
\hline
\end{tabular}

\section{4 结论}

（1）三维多孔点阵结构在与柔性树脂这种软性 材料的相互结合下展现出了良好的力学特点。其在 轴向压缩下, 共经历了弹性形变, 弹性屈曲和致密 化的三个变形阶段。

（2）由于这是一种微小型的多孔结构, 所以其 内部结构和尺寸参数都是影响其压缩性能的重要特 征。根据本文的研究发现, 在相同结构的情况下, 杆件直径越大, 所表现出来的承载能力就越大, 平 台应力值越大, 但杆件直径越大, 其孔隙率就越小, 且相对密度越大，可压缩空间就会变小，导致致密 化过早, 从而在应力-应变曲线中所表现出过早进入 致密化效应段，曲线的斜率迅速上升。

（3）在相同杆件直径的不同结构条件下，单胞 3 所组成的点阵结构 3 所表现出的压缩性能以及压力 平台最为良好，在相同的加载条件下，其平台应力 值和吸能性能都为最优。

（4）通过改变结构的点阵层数以及不同的排列 方式发现，其表现出来的压缩性能特点也截然不同。 三层结构与双层结构相比, 其压缩应力平台更宽, 平台应力值也较双层的高。但劣势就在于其整体厚 度过大，在一些较薄件填充时并不适用。

综上, 本文研究结果为多孔材料的吸能特性的 优化设计提供了新的思路。

在此, 强调非常重要的一点, 该三维多孔点阵 结构是一种可控的有规律的三维结构, 并不像蜂窝 状的一种随机的多孔结构。本文设想在后期的研究 中，通过这一种可控的多孔结构，精确控制其相对 密度进行梯度设计; 将几何参数或者材料参数沿多 孔点阵结构厚度方向进行梯度设计。但由于层数的 增加使得整体厚度过大，因此需要探讨胞体杆件与 $Z$ 轴所成的夹角对结构压缩性能的影响, 在保证一 
定厚度的情况下研究其夹角对整体结构压缩性能的 影响, 最终对结构的吸能平台以及压缩力学性能做 进一步的优化。同时也对该结构进行拉伸、剪切等 力学性能的研究。

\section{参 考 文 献}

[1] SIGMUND O. Tailoring materials with prescribed elastic properties[J]. Mechanics of Materials, 1995, 20(4): 351-368.

[2] 敬霖, 赵永翔. 轻量化多孔金属夹芯复合板的耐撞性 $[\mathrm{J}]$. 机械工程学报, 2014, 50(16): 1-5.

JING Lin, ZHAO Yongxiang. Crashworthiness of light-weight sandwich panels with cellular metallic cores [J]. Journal of Mechanical Engineering, 2014, 50(16): $1-5$.

[3] 曾嵩, 朱荣, 姜炜. 金属点阵材料的研究进展 [J]. 材料 导报, 2012(5): 18-23.

ZENG Song, ZHU Rong, JIANG Wei. Research progress of metal lattice materials[J]. Materials Reports, 2012(5): 18-23.

[4] LIM C H, JEON I, KANG K J. A new type of sandwich panel with periodic cellular metal cores and its mechanical performances[J]. Materials \& Design, 2009, 30(8): 3082-3093.

[5] QUEHEILlALT D T, WADLEY H N G. Cellular metal lattices with hollow trusses[J]. Acta Materialia, 2005, 53(2): 303-313.

[6] DESHPANDE V S, FLECK N A. Collapse of truss core sandwich beams in 3-point bending[J]. International Journal of Solids \& Structures, 2001, 38(36-37) : 6275-6305.

[7] WADLEY H, DHARMASENA K, CHEN Y C, et al. Compressive response of multilayered pyramidal lattices during underwater shock loading[J]. International Journal of Impact Engineering，2008，35: 1102-1114.

[8] 范华林, 杨卫, 方岱宁. 新型碳纤维点阵复合材料技术 研究[J]. 航空材料学报, 2007, 27(1): 46-50.

FAN Hualing, YANG Wei, FANG Daining, et al. Interlacing technique for new carbon fiber lattice material[J]. Journal of Aeronautical Materials, 2007, 27
(1): 46-50.

[9] 柏龙, 熊飞, 陈晓红. SLM 制备的 Ti6Al4V 轻质点阵结 构多目标结构优化设计研究[J]. 机械工程学报, 2018, 54(5): 156-165.

BAI Long, XIONG Fei, CHEN Xiaohong. Multi-objective structural optimization design of Ti6Al4V lattice structure formed by SLM[J]. Journal of Mechanical Engineering, 2018, 54(5): 156-165.

[10] 卢天健, 徐峰. 皮肤的力学性能概述 [J]. 力学进展, 2008, 38(4): 393-426.

LU Tianjian, XU Feng. Mechanical properties of skin: A review[J]. Advances in Mechanics, 2008, 38(4) : 393-426.

[11] ZHU X, CHEN Y, LIU Y, et al. Additive manufacturing of elastomeric foam with cell unit design for broadening compressive stress plateau[J]. Rapid Prototyping Journal, 2018, 24(9): 1579-1585.

[12] 张征, 吴化平, 李祥辉, 等. 金字塔点阵复合材料结构 力学性能分析与优化 [J]. 轻工机械, 2013, 31(1): 74-79. ZHANG Zheng, WU Huaping, LI Xianghui, et al. Mechanical properties analysis and optimal design of pyramid lattice composite structures[J]. Light Industry Machinery, 2013, 31(1): 74-79.

[13] 易长炎, 柏龙, 陈晓红, 等. 金属三维点阵结构拓扑构 型研究及应用现状综述[J]. 功能材料，2017，48(10): 10055-10065.

YI Changyan, BAI Long, CHEN Xiaohong, et al. Review on the metal three-dimensional lattice topology configuration[J]. Journal of Functional Materials, 2017, 48(10): 10055-10065.

[14] 刘鸠. 活体状态下人脸皮肤力学性能试验研究[D]. 天 津: 天津大学, 2017.

LIU Peng. Experimental study on the mechanical properties of human face skin in a living state[D]. Tianjin: Tianjin University, 2017.

作者简介: 纪小刚(通信作者), 男, 1977 年出生, 博士, 副教授, 硕士 研究生导师。主要研究方向为逆向工程、计算机辅助设计技, 快速原型 技术。

E-mail: bhearts@126.com

张建安, 男, 1996 年出生。主要研究方向为多孔点阵结构。

E-mail: 1516123056@qq.com 